\title{
A Novel Method for Teaching Knowledge about Binary System
}

\author{
Hui Xie, Xiaoying Wang, Lihua Fan and Xiaojing Liu \\ Department of Computer Technology and Applications, Qinghai University, Xining, Qinghai 810016, China \\ xdhui1981@163.com
}

\begin{abstract}
Binary system and relative knowledge alway are considered as an abstract and hard point in the introducing IT courses to the students just start learning IT. We present a novel method using an analogy based on shelf that could teaching these knowledges vividly and efficiently. The evaluation show our method could advance the teaching process about Binary and worthed to be referenced by teachers when they face the problem how to teaching number system and binary system
\end{abstract} analogy

Index Terms - number system, binary system, teaching,

\section{Introduction}

While Binary system just like a door to the world of information technology, knowledge about binary system is one of the most essential and important parts in the introductory course for a beginner who want travel the IT world. Meanwhile, binary system is so different to the traditional number system used in our life, and also it is very abstract, academic and professional, so binary system has become a knowledge point which is very difficult to understand for new travelers. It is believed that most of the students who is in the initial stage for learning IT courses have confused or dropped into a strait when encountering the binary system. Some even lost they interests or give up in studying these courses. We once did a questionnaire survey to non-ITmajor students who have finished basic IT courses in our school, the statistical result show that $85 \%$ of the questionnaire agree binary system is hard to understand, and $50 \%$ of them express they still have been being puzzled by it and $30 \%$ have no idea about it. The result is more frustrating in our social education classes. Of course, students in IT-major show a good result about Binary system studying, but they still tell us that they cost a lot of time to drill it. Undoubtedly, binary system has bring many difficulties to both the universal education and professional education for IT.

In fact, it is not something very abstruse and mysterious just like the theory of Einstein's theory or Goldbach's conjecture, the content of binary system is clear and definite. Binary system appear just a little strange and abstract, and the difficulty for understand it always go into effect to beginner, when you once comprehend it, you will exclaim "So that is what it is". Thus, we need some teaching technics or methods to help students to understand the point easily and quickly.

How could we let the process of teaching or learning binary system become easier, simpler, more vivid and efficient? What should we do to let the students understand the binary system quickly and deeply in a comfortable way?
Paper [1] using history cases to introduce the number system and data representation, but cannot bring a deep and systemic understand to student about binary. Because students in the paper is schoolchild, the teaching goal just is giving a simple cognize about binary .Zhang Li-bo [2] introduce a way using a guessing number game to teaching convert between decimal and binary numbers, which could draw the students' interests and help them to achieve conversion, but lacks efficient introducing method about binary and may cost much time. JIANG Wan-jun et.al. [3] discussed a method to realize conversion between decimal and binary in a fast way, but this paper only tells an skill to students without giving deep and systemic understanding about binary. Wang Xia-cheng et.al.[4] present the common theory and method for conversion between different number systems, but it is too academic and lacks vivid introducing. Cui Yong-zhi [5] used turning on and off a line of bulbs to introduce binary system, it seems to be some interesting and bring some help to student to learn the binary, but this method still lacks deep and his conversion process is too dull to learn.

We have been thinking these questions for a long time, and keeping to design, improve, practice method and technic again and again, we get a novel and efficient method for teaching/learning the binary system. The rest of this paper will present this method in detail.

\section{Introduction}

Although people use number system in their real life every day, most of them do not have a scientific and accurate understand, as a result, many students will question what exactly a number system is, and they will ask why the binary system is used in a computer system. For eliminating these question and introduce the binary system smoothly, we designed a teaching flow for our class about binary system as Fig. 1.

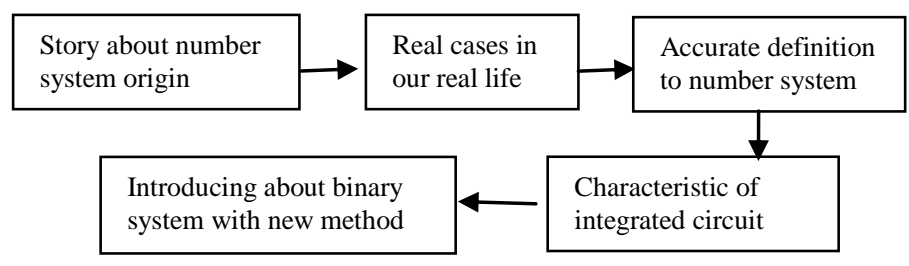

Fig. 1 Teaching flow about binary system 
Frist ,we use the story about primitive's food collecting and counting trouble as the start of class, tell student the origin about number system and importance for human.

Secondly, we use the cases of number system, such as weekday to septenary, 12 months constitute a year to the duodecimal, clock to the sexagesimal, etc. and let students experience and think the number system.

Thirdly, give the accurate definition about number system(some student may still confused, which doesn't matter, since our novel analogy in the later of this paper may help them to understand ).

Fourthly, introduce the characteristic of integrated circuit and computer system, tell students the reason for using binary system.

At last, and the main part of class, use an analogy based on shelf to introduce what is binary system.

\section{Analogy between Number System and Shelf}

This section is the kernel of the paper. Here we will introduce the most valuable wealth and also the innovation of our method- a analogy between number system and a good shelf we could call it Shelf-Analogy. For a more vivid effect and attracting students' interesting and initiative, we design a virtual scene base on our novel Shelf-Analogy.

\section{A. Analogy between decimal system and shelf}

Decimal system could be explained by the following virtual scene.

Imagined scene 1: Tom get a part-time job during the summer vacation in a supermarket. The mission of tom's job is managing a shelf for fruit cans specially (Fig. 1). These cans must be put on the shelf by the following rules:

(1) the first floor of the shelf is just only used to place cans in bulk, and the maximum of cans could be put on the first floor is 9 , if 10 cans appear on the floor ,they must be packed into a box called A-box and put it on the up floor right now;

(2) the second floor is required to place A-box( contain 10 cans) only. The number of A-box could be put on the this floor range from 0 to 9 . whenever there are $10 \mathrm{~A}$-box on this floor, these 10 A-box must be packed into a bigger box called B-box and put it on the third floor immediately.

(3) just like the under two floor, the third floor abide the convention that objects could be put on it in the range $0-9$, and objects must be packed in a C-box if there are 10 objects collected ,then put this C-box to the fourth floor immediately . Certainly, the object could be put on the third floor is only Bbox .

(4) the fourth floor is the end of the shelf, it just accept C-box only. On this floor, there are 9 B-box at most. Because this is the last floor, there has no need for packing box.

If Tom could do this job well, he may earn $100 \$$ per week. Could you do this job like Tom? Of course, if you can, besides dollars you earn, you also know the fact of decimal system too. The shelf in the Imagined scene 1 is just a demo for decimal system.
Figure 2 shows the result for put 1126 cans to a good shelf use rows above, and relationship between the result and corresponding Decimal number.

In addition, we give the concept of weight in our paper. The weight indicate the capacity to contain cans in-bulk of a basic unit on the floor. For example, a can's weight is $10^{\circ}$, Abox's weight is $10^{1}$, B-box's weight is $10^{2}$,C-box's weight is $10^{3}$,etc. Now, the loaded shelf in Fig. 2 contains $1 * 10^{3}$ $+1 * 10^{2}+2 * 10^{1}+6^{*} 10^{0}=1126$, corresponding to the number of the cans.

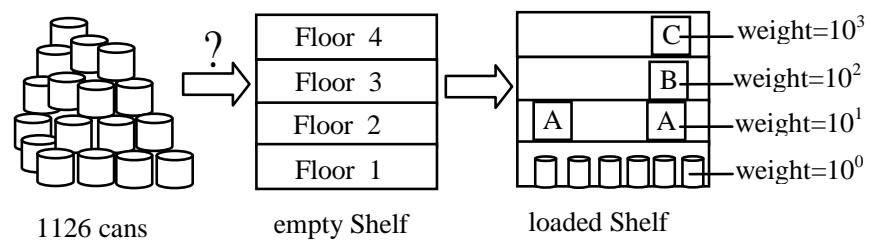

Fig. 2 put 1126 cans to shelf use rows above

\section{B. Analogy between Binary system and shelf}

Benefited from the face that human are accustomed to Decimal system, we could easily understand the analogy between decimal system and shelf, know the essence of decimal system. Now, we could introduce the Binary system smoothly based on the Imagined scene 1 just do a little changes in the rules .Changes for rules:

(1)Max-number of objects could be placed on the first floor is 1 .it means there is only 0 or 1 object on every floor.

(2)Whenever the number of the objects on the floor up to 2 , these 2 objects must be packed in a more lager box and put it to the upstairs floor immediately.

Imagined scene 2: Because Tom do well in his part-time job for managing the shelf, he is arranged to manage one more shelf, of course he is paid more dollars. The new one shelf has 4 floors the same, but asked be managed under new rules (originated from Imagined scene 1 and changed by changes above) just like the following:

(1) the first floor of the shelf is just only used to place cans in bulk, and could have 0 or 1 can at most, if there are 2 cans ,they must be packed into a box called X-box and put it on the upstairs floor at once;

(2) the second floor is required to place X-box( contain 2 cans) only. The number of X-box could be put on the this floor range from 0 to 1 . whenever there are $2 \mathrm{x}$-box here, these $2 \mathrm{X}$-box must be packed into a bigger box called Y-box and put it on the third floor promptly.

(3) only Y-box could be put on the third floor, box number is 0 or 1.packing will happen when $2 \mathrm{Y}$-box appear on this floor, and the box produced called Z-box (contain $2 \mathrm{Y}$ box).of cause the Z-box should be put on the fourth floor immediately.

(4) the fourth floor is the end of the shelf, it just accept Zbox only. On this floor, there may have 0 or $1 \mathrm{Z}$-box. Because this is the last floor, there is no packing will happen. 


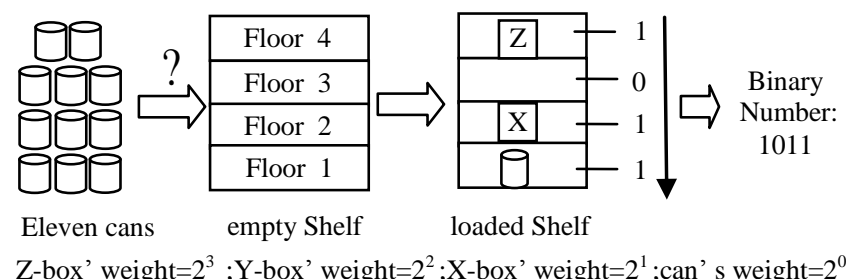

Fig. 3 put eleven cans to shelf use rows in scene 2

Just like play game, if you could do well in previous scene 1 ,you may do well in this scene obey the cans-place rules above.

Figure 3 shows result for put eleven cans on a Binarysystem shelf and the comparison between the result and corresponding Binary-system number.

\section{Other number System}

Now you may have an intuitive recognition and deep understand about Decimal and Binary system through the analogy base on shelf.

We could use similar analogies to introduce octonary and hexadecimal system which are also two common number systems in IT courses. Of course we can use similar analogies to introduce other number System such as 7-,12-,60- system, etc.

\section{Instruction about Number System Convert using Shelf- Analogy}

There are many reasons for needing convert between different number systems in many situation in IT area. So number systems convert is an essential knowledge point about Binary system in IT introductory courses, students always be required to understand the principle for convert and could carry out a given number's convert between two different systems. Unfortunately, this point is a hard point in the lesson about binary system for new guys who want learn IT courses. According to our statistics result for 3 years' score status about number systems convert on paper for the course of 'computer application basic' in our college, there are only $65 \%$ of the examinee got the point on average. It believed the score rate is lower in adult education and universal education about IT or computer application.

In this section, we will discuss how to introduce number systems convert using our Shelf-Analogy.

\section{A. Convert between Decimal and Binary System}

\section{1) Decimal to Binary}

Many books [6][7] give the method for Decimal to Binary. it always be presented as following:

To convert from a base-10 integer numeral to its base-2 (binary) equivalent, the number is divided by two, and the remainder is the least-significant bit. The (integer) result is again divided by two, its remainder is the next least significant bit. This process repeats until the quotient becomes zero [8].As an illustration, figures similar to the block surrounded by dotted line in Figure 5 be used normally.
Though these presents give the accurate describe for decimal to binary, they are not intuitive and vivid enough, and even too abstruse and dull to the freshmen in introductory course about IT. They often doubt why be divided by two? why bottom up the remainders produced in sequence?

We use Shelf-Analogy to reveal the convert process, answer why divided by 2 and why bottom up the remainders. Actually, the process putting eleven cans to a binary-shelf in figure 3 is a typical case for decimal to binary. The substance of decimal to binary is just like how to place cans counted by decimal to a binary-shelf.

Figure 4 show the detail of how to place eleven cans to the binary-shelf. The key point is the packing step, and all packing operation must obey the rules in Section 3.
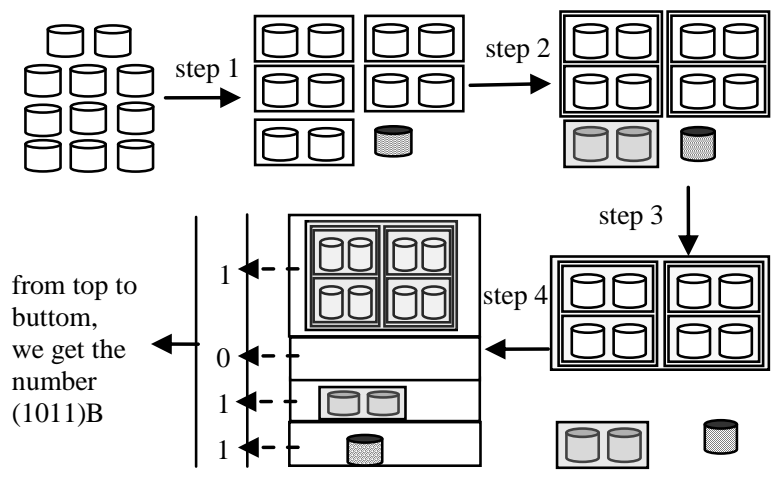

Fig.4 detail of how to place eleven cans to the binary-shelf

In the first step, we pack all eleven cans in-bulk to $\mathrm{X}$ box(contain 2 cans). The way is circulate the process which take two cans from the all the in-bulk cans rest and pack into a $\mathrm{X}$-box until there is no more than 1 can left. The result is $5 \mathrm{X}$ boxes and 1 in-bulk left, the nature of this step equals he mathematical process that eleven is divided by two, the result is 5 and the remainder is 1 , here 5 indicate the number of $\mathrm{X}$ boxes produced and remainder 1 represent the one can left.

The second step, to pack X-box to Y-box (contain $2 \mathrm{X}$ boxes).The process is similar to step 1,the different is the object to be packed is $\mathrm{X}$-box, and the product is Y-box, and the rest is 0 or $1 \mathrm{X}$-box. Using the rules described before, the 5 $\mathrm{X}$-box produced by step 1 will be packed and produce $2 \mathrm{Y}$ box, and $1 \mathrm{X}$-box will left. This process equals the process for dividing 5 by 2 , the result 2 indicate 2 Y-box produced and remainder 1 represent the left $1 \mathrm{X}$-box.

The third step, to pack Y-box to Z-box. The process for packing $2 \mathrm{Y}$-box is just to put the $2 \mathrm{Y}$-box into one Z-box and close it, just equal to divide 2 by 2, 0 is left.

The fourth step, keeping packing, trying to pack Z-box to more lager box(contain 2 Z-box).in this process, because only 1 Z-box exit, so we could not go on packing, all packing work is end. This step equal the process divide 1 by 2 , the result is 0 , and the remainder is 1 .

The all 4 steps could be represented by a mathematical process as Figure 5. 


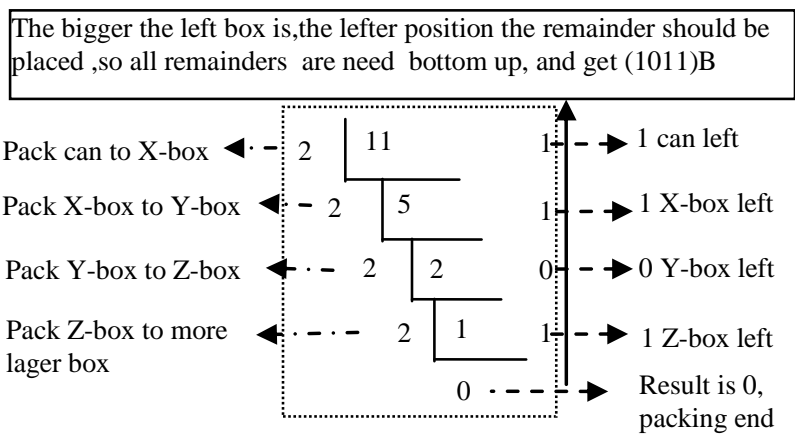

Fig.5 put eleven cans to shelf use rows in scene 2

Note that it could not go on packing correspond to the result of dividing is 0 , this is the signal to end packing.

We could find that the later the remainder appear, the lagger the box represent by the remainder is. Now ,the remainders of step 1-4 in sequence is:1,1,0,1.They represent objects is 1 can, 1 X-box, 0 Y-box, 1 Z-box. Because the position's power in a number is decrease from left to right(corresponding high floor to low floor in binary shelf ), we need bottom up the remainders sequence to express the number correctly and the ultimate result for all the 4 steps above is 1011 .

With this packing process for eleven to (1011)B, we could easily give students a intuitive and clear introduce about Decimal to Binary convert.

2) Binary to Decimal

This question is relative simple to the Decimal to Binary one. we will use figure 6 to illustrate and a brief words to explain it. Figure 6 shows the process for how to convert Binary number (1011)B to decimal. The essence of the process is to count all the cans in shelf, despite in or no in a box. Cans in any one floor could be counted by the formula:

$$
t_{i}=n_{i}^{*} w_{i}
$$

here $\mathrm{t}_{\mathrm{i}}$ represent the total number of cans on the floor $i, n_{i}$ is the number of objects(either appropriate box or in-bulk cans ) in this floor, $w_{i}$ represent the weight of the object. By the way, the weight for every floor's object could be inferred by formula:

$$
w_{i}=2^{i-1}
$$

here $i$ indicates the floor number. Finally, the total number $\mathrm{T}$ on the while shelf is:

$$
\mathrm{T}=\sum_{i=1}^{m} t_{i}=\sum_{i=1}^{m} n_{i} * w_{i}=\sum_{i=1}^{m} n_{i} * 2^{i-1}
$$

According to (3), the total number of cans in figure 5 could be inferred as follow:

$$
T=1 * 2^{4-1}+0 * 2^{3-1}+1 * 2^{2-1}+1 * 2^{1-1}=8+0+2+1=(11) D
$$

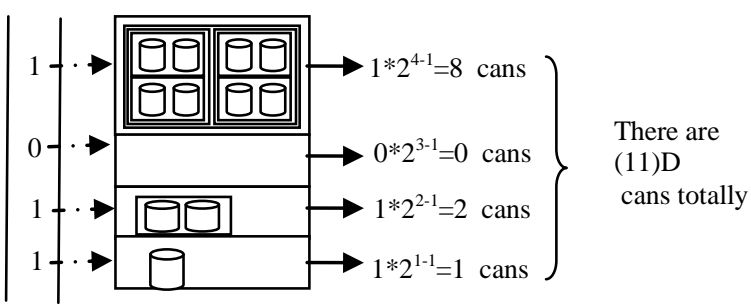

Fig.6 process for convert Binary number (1011)B to Decimal

In addition, we can infer the other number system such as octonary, hexadecimal convert to Decimal using the similar process just like Figure 5, with changing $\mathrm{w}=2^{\mathrm{m}-1}$ to $\mathrm{w}=8^{\mathrm{m}-1}$ or $\mathrm{w}=16^{\mathrm{m}-1}$.

\section{B. Convert between Binary and Octonary System}

Well, we can use the decimal as the bridge to achieve converts between octonary and binary. But there is a more effective and direct way to convert them. This way is always introduced in many books too, but sometimes their introduces seem to be too sketchy to let students know why could use this way execute convert between octonary and binary directly. We use the shelf to introduce this point giving students an intuitive feel about it.

Figure 7 show a comparison between an Octonary-floor and three Binary-floor. On the octonary-floor we can put 0-7 cans, and on the three Binary-floor we can put 0-7 cans too.it show an octonary-floor equals to three Binary-floor corresponding. In other words, a position in an octonary corresponding to 3 positions in an binary. Of course, we have to state that align right is required here to the convert, it means we process the potions from right to left.

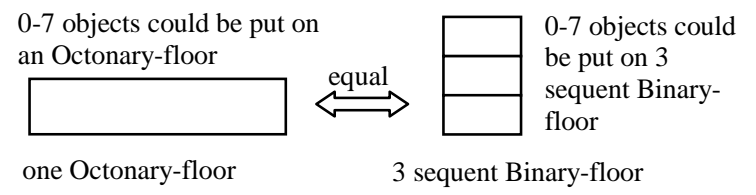

Fig.7 comparison between an Octonary-floor and three Binary-floor

Figure 8 shows an example for converts between octonary and binary directly for the number (6207)O. We can use the similar way to achieve converts between hexadecimal and ninary directly. Due to the constraints of the length of this paper, we will not discuss it here.

\begin{tabular}{|ccccc}
\hline Octonary & 6 & 2 & 0 & 7 \\
\hline 1 & 1 & 1 & 1 \\
\hline Binary & $\vdots$ & 1 & $\vdots$ & $\vdots$ \\
\hline 110 & 010 & 000 & 111 \\
\hline
\end{tabular}

Fig. 8 an example for converts between Octonary and Binary directly

\section{Evaluation of our Teaching Flow and Analogy based on Shelf}

For Evaluate the effect of the method for introducing Binary system with our teaching flow and Analogy based on shelf, we chose 10 different classes which just start learning 
the course of Computer Application Basis from different major in our college. There are 5 classes were taught with method teacher used before, and the other 5 classes were introduced the binary system with the new teaching flow and method we present in this paper. After the class, we tested students with paper about binary system knowledges, and take an investigate with questionnaire for status about " do you understand? is easy or hard to learn? is dull or interesting ?" etc. We still statistics the time consumed by the process for teaching ninary system. And then we combine and normalized the statistics data. Table I is the main data for evaluation.

TABLE I Main statistics data for Evaluation

\begin{tabular}{|c|c|c|c|c|}
\hline & $\begin{array}{c}\text { Time needed } \\
(\mathrm{min})\end{array}$ & Understand & $\begin{array}{c}\text { Score } \\
\text { rate }\end{array}$ & $\begin{array}{c}\text { Feel Easy } \\
\text { to learn }\end{array}$ \\
\hline old method & $15-35$ & $65 \%$ & $60 \%$ & $50 \%$ \\
\hline new method & $10-20$ & $90 \%$ & $95 \%$ & $95 \%$ \\
\hline
\end{tabular}

According to Table I, it shows that our method presented in paper could raise the effect and cut down the cost for teaching knowledge about binary system.

\section{Conclusions}

We present a novel method to teaching Binary system and relative knowledges in the IT introducing course for beginner. the valuable innovate of this paper is using an Analogy based on shelf to explain the essence and the relationship about number system, especially for Binary system. the result of evaluation show the our method could get an good effect compare to the traditional method. and this method worthed to be referenced by teachers when they face the problem how to teaching number system and binary system.

\section{Acknowledgment}

This paper is partly supported by the High Education Research and Reform Project in Qinghai Province ("Implementation and Application of Home-work Similarity Detection Tools") and also the Course Construction Project in Qinghai University (No. KC-13-1-1).We would like to thanks for teams of computer basic teaching give many support and accompany us with whole study process. we would also thanks students in the chosen 10 classes, they cooperate with us well to do the investigate by questionnaire.

\section{References}

[1] Li.Xuzahng. Teaching "The Number System and Data Representation"Base on the Historical Development. COMPUTER EDUCATION, no.22,pp.39-42,November 2009

[2] Zhang Libo. Teaching "convert between Decimal and Binary" with a Guessing number game. CHINA EDUCATIONAL TECHNOLOGY, no3, pp95-97, March 2008

[3] Jiang Wan-jun. Discuss teaching for binary system. JOURNAL OF SCIENCE OF TEACHERS' COLLEGE AND UNIVERSITY, vol.27, no.2, pp78-80, February 2007.

[4] Wang Xia-cheng. Discuss for teaching method about convert between different number system. Management \& Technology of SME.no.36,pp 265-266,December 2013.

[5] Cui yong-zhi. Study of teaching method about binary system. JOURNAL OF HEXI UNIVERSITY. vol 20,no2, pp10-13,February 2004.

[6] Jiang Li-rong. collage computer basics. PEKING UNIVERSITY PRESS. 2008

[7] $\mathrm{Ma} \mathrm{Li}$, collage computer basics. SOUTHEAST UNIVERSITY PRESS. 2006

[8] http://en.wikipedia.org/wiki/Binary_number 